(2) Open Access Full Text Article

REVIEW

\title{
Improving drug abuse treatment delivery through adoption of harmonized electronic health record systems
}

This article was published in the following Dove Press journal:

Substance Abuse and Rehabilitation

19 July 201 I

Number of times this article has been viewed

Udi E Ghitza

Steven Sparenborg

Betty Tai

Center for the Clinical Trials Network, National Institute on Drug Abuse, National Institutes of Health, United States Department of Health and Human Services, Bethesda, MD, USA
Correspondence: Udi E Ghitza Center for the Clinical Trials Network, National Institute on Drug Abuse, National Institutes of Health, 6001 Executive Boulevard, Bethesda, MD 20892, USA

Tel +I 30I 4436697

Fax + I 30I 44323 I7

Email ghitzau@nida.nih.gov
Abstract: A great divide currently exists between mainstream health care and specialty substance use disorders (SUD) treatment, concerning the coordination of care and sharing of medical information. Improving the coordination of SUD treatment with other disciplines of medicine will benefit SUD patients. The development and use of harmonized electronic health record systems (EHR) containing standardized person-level information will enable improved coordination of healthcare services. We attempt here to illuminate the urgent public health need to develop and implement at the national level harmonized EHR including data fields containing standardized vocabulary/terminologies relevant to SUD treatment. The many advantages and barriers to harmonized EHR implementation in SUD treatment service groups, and pathways to their successful implementation, are also discussed. As the US Federal Government incentivizes Medicare and Medicaid Service providers nationwide for "meaningful use" of health information technology (HIT) systems, relevant stakeholders may face relatively large and time-consuming processes to conform their local practices to meet the federal government's "meaningful use" criteria unless they proactively implement data standards and elements consistent with those criteria. Incorporating consensus-based common data elements and standards relevant to SUD screening, diagnosis, and treatment into the federal government's "meaningful use" criteria is an essential first step to develop necessary infrastructure for effective coordination of HIT systems among SUD treatment and other healthcare service providers to promote collaborative-care implementation of cost-effective, evidence-based treatments and to support program evaluations.

Keywords: electronic health records, substance use disorders treatment, addiction, dependence, research, Clinical Trials Network

\section{Introduction}

There is an urgent public health need to nationally develop and deploy harmonized electronic health record systems (EHR) containing standardized vocabulary/ terminologies that enable comprehensive coordinated patient care. Harmonized EHR refers to systems using consistent standards enabling electronic communication and information sharing with one another across networks. In a recent national survey of US acute care hospitals on the presence of specific electronic-record functionalities, very few $(<2 \%)$ hospitals were found to have a comprehensive electronic system for recording clinical information, and only a small minority (between $8 \%$ and $12 \%$ ) have even a basic system. ${ }^{1}$ The authors conclude that the low prevalence of harmonized EHR systems nationally in US hospitals precludes an easy exchange of clinical data between hospitals or from hospitals to 
physicians' offices. Furthermore, fragmentation of the US health IT system has meant that decision-making on health IT investment is usually a local decision predicated upon local needs, rendering health delivery coordination, often difficult due to a lack of generally accepted national data standards and terminologies/vocabularies. ${ }^{2}$ The need to improve coordination of health care nationally is apparent across all disciplines of medicine, but nowhere is this more urgent than coordinating illicit drug, alcohol, and tobacco use disorder treatments and mainstream medical services such as emergency, primary, and specialty care providers. The current, fragmented behavioral health treatment comes about because public SUD and mental health treatment is typically not fully coordinated with the rest of the US healthcare system. Consequently, there is a great divide between mainstream health care and specialty SUD treatment. ${ }^{3,4}$ Mainstream health care is now pursuing the adoption of harmonized EHR to facilitate implementation of care within itself. In order to coordinate SUD treatment with mainstream health care services, specialty SUD treatment systems must implement EHRs that are interoperable with those of mainstream health care. An essential first step for SUD treatment providers is to develop standardized common data elements relevant to SUD patients and treatment (including questions on alcohol and tobacco use/ misuse) to be incorporated into harmonized EHR. This is especially true because, to date, harmonized EHR containing common data elements with standardized vocabularies relevant to SUD treatment are not used consistently among community-based treatment programs.

The current widespread misuse and abuse of prescription opioid medications in the US, which has increased dramatically in recent years, ${ }^{5,6}$ means that even if only a small percentage of patients are vulnerable to misuse and abuse of prescription opioids, physicians still need to screen for substance use disorders in order to provide adequate and appropriate care. A major benefit to be derived from use of the EHR containing standardized common data elements relevant to SUD treatment is to enhance the collaboration between physicians and pharmacists to reduce the likelihood of unsafe drug-drug interactions, overdose incidents and deaths, and, in so doing, better serving the goals of prescription drug monitoring programs to reduce such serious adverse events and enhance patient safety. However, these important patient-safety measures could not be taken if alcohol and non-prescribed drugs used by patients are not recorded on the EHR and consulted by both physicians and pharmacists when new medications are prescribed.
The many advantages and barriers for implementing EHR containing standardized data elements in these behavioral health service groups, and pathways to their successful implementation, are discussed below.

\section{Advantages of interoperable electronic health record systems}

The development of digitized medical records dates back at least two decades. In a 1991 Institute of Medicine report, development of computerized patient records was considered a key infrastructural requirement for supporting national health information management needs. ${ }^{7}$ Subsequently, the US Congress mandated the National Library of Medicine, National Institutes of Health to issue a Broad Agency Announcement to support research and development to explore the barriers and utility of health information technology (HIT) for healthcare applications. ${ }^{8}$ A type of record that fulfills the congressional vision for shared information is known today as an Electronic Health Record (EHR), defined by The National Alliance for Health Information Technology as an "aggregated electronic record of health-related information on an individual that is created and gathered cumulatively across more than one health care organization and is managed and consulted by licensed clinicians and staff involved in the individual's health and care".

Data interoperability, a key infrastructural feature of functional EHR, is the ability of separately owned and managed information systems to use consistent standards enabling electronic communication and information sharing with one another across networks. ${ }^{9}$ Interoperable EHR may promote the implementation of cost-effective, evidence-based treatments and support treatment program evaluations that will determine best patient-care practices leading to improved patient outcomes. ${ }^{10-12}$ Furthermore, EHR may facilitate treatment research significantly, by allowing real-time on-site data capture, and convenient two-way data flow between researchers and clinicians. Interoperable EHR may also enable large-scale, longitudinal population-based health research assessing the impact of healthcare approaches over time. Such analyses may effectively complement randomized controlled trials in the development and adoption of evidence-based medicine to manage chronic disease conditions, ${ }^{11-13}$ such as diabetes, hypertension and SUD. Furthermore, interoperable EHR enable providers to automate, structure, and streamline clinical workflow among various disciplines resulting in improved efficiency of data-based medical decision-making processes. 
Three critical elements of functionally interoperable EHR are:

1. The ability of these systems to collect and store patient data electronically, such as demographics, patient problem lists (eg, diagnoses, medications, allergies, notes, and summaries), including longitudinal collection of information (such as a history of the patient's medical conditions);

2. The ability to make these data available to multidisciplinary providers upon request, after applying privacy and security controls consistent with stipulations of the Health Insurance Portability and Accountability Act (HIPAA) and CFR 42 Part 2;

3. The ability to provide clinicians with computerized decision support tools - that is, to present them with standard, evidence-based options for medical decision making regarding individual patients. ${ }^{12}$

\section{Barriers to implementation}

Progress in developing and applying interoperable EHR in the past 2 decades has been marginal due to major barriers. ${ }^{1,14-16}$ A recent national survey of physicians found that only $4 \%$ of physicians report having extensive, fully functional EHR, and only $13 \%$ reported having a basic system. ${ }^{16}$

A major barrier to wide implementation of EHR in all disciplines of health care is the lack of interoperability. The data architecture and vocabulary of currently available EHR often resemble silos, which are unable to exchange key data elements with one another. A critical prerequisite step to implement interoperable behavioral health EHR, in the context of community-based SUD treatment, is for all stakeholders (for example, providers, state regulatory officials, payers, consumer representatives, and SUD treatment researchers) to decide upon a core set of common data elements and common standardized tools relevant to SUD patient screening, assessment, and treatment. For instance, state agencies may require inclusion of the 7 domains of the Addiction Severity Index (ASI) while JCAHO-accredited agencies may require inclusion of 6 dimensions of assessment of the American Society of Addiction Medicine (ASAM) PPC tool. These tools capture similar information with dissimilar vocabularies/terminologies and time frames. Also, physician order entries may differ in format from those order entries written by other clinicians. These need to be standardized. SUD treatment providers should be asked to collect a standardized set of core data elements relevant to treatment. This action may minimize inconsistency, redundancy, and inefficiency leading to improved communication among behavioral health care providers.

Specific additional challenges facing the SUD treatment communities include:

1. Technological and infrastructural challenges: Many agencies do not yet have decent access to well-developed health information/data systems. ${ }^{17,18}$ HIT sophistication and computer literacy in SUD treatment programs varies greatly. Additionally, SUD treatment settings vary widely in approach, goals, and size from inpatient detoxification to intensive drug-free outpatient care, to residential treatment, to office-based opiate replacement therapy settings, and so on. As a result of this wide variability and lack of capability for health information exchange (HIE), some approaches to SUD treatment and the application of EHR in these diverse clinical settings may be less amenable to database-derived structure than in other medical practices. In an exploratory study, Wisdom et al found that no SUD treatment agencies in their sample have capability for HIE, to communicate electronically across treatment programs. ${ }^{18}$

2. Regulatory challenges: Largely due to concern about federal privacy laws, SUD treatment providers - even those using EHR - thus far have mostly been excluded by healthcare organizations already exchanging patient data. The federal confidentiality regulations drafted in the early 1970 s, commonly referred to as 42 CFR Part 2, state that without specific authorization in the form of written consent from the patient about what information may be disclosed (as specified by 42 CFR 2.31), health care providers cannot routinely access patients' substance use history and current treatment regimen, except in a medical emergency (42 CFR 2.51). ${ }^{19}$ This prohibition on unauthorized re-disclosure is an issue for policymakers developing consent regulations for exchange of EHR information. Also complicating matters, some state regulations provide conflicting or even more stringent guidelines on protecting sensitive health information.

Currently, there is a controversy on whether to modify this law to allow, in the electronic age, health care providers free access to the EHR as part of integrated care and interoperable health information. Coalition groups led by Attorney Renée Popovits and Eric Goplerud, PhD, a research professor in the Department of Health Policy at George Washington University, advocate that the US Congress should modify 42 CFR Part 2 to ensure that SUD treatment organizations could participate in health information exchanges. While stressing that statutory 
and regulatory language must continue to protect patient rights and prohibit discrimination, these individuals maintain that the sharing of substance use information for specific purposes among entities covered by the HIPAA is key to providing effective health care. ${ }^{19}$ Others, represented by patient advocacy groups like Faces and Voices of Recovery, the Legal Action Center (LAC), and Carol McDaid, principal with Capitol Decisions, Inc, have declined to endorse the proposal to change the law, although they recognize that EHR would improve patient care. ${ }^{20}$ Instead, the LAC position paper states that the current federal laws "are not a major barrier" to integrating SUD treatment services with the rest of the healthcare system, and that the goal of improving health communication should focus on tweaking the federal government's interpretation of the existing law. ${ }^{20}$

There is also a risk that mandating common data elements pertinent to screening, diagnosis, and treatment of SUD in interoperable EHR and thus sharing of diagnostic information, may dissuade some patients from honest reporting or treatment seeking altogether. ${ }^{21,22}$ Notably, this risk could be managed and reduced in several ways.

First, a possible resolution of the privacy issue regarding CFR 42 Part 2 and related information sharing concerning screening and diagnostic information relevant to SUD is data segmentation, which supports granular selection of disclosing, accessing, and utilizing health information regarding specifying which provider has access to patients' health information and under which circumstances, by which period of time, and by whom this information is to be exchanged. Substance Abuse and Mental Health Services Administration (SAMHSA) is currently working with DHHS Office of the National Coordinator for Health Information Technology (ONC) and other pertinent federal agencies to create privacy policies to support patient consent in using data segmentation through the DHHS ONC Nationwide Health Information Network-CONNECT gateway.

Second, in concert with the data segmentation approach mentioned above, the use of physician portals, which permit outside providers to access EHR records through a web-based portal, may offer an opportunity for the sharing of health information among SUD treatment and other service providers. The capability of SUD treatment providers to access the physician portal in primary care EHR systems would help facilitate admissions in specialty SUD inpatient and outpatient treatment programs, as these providers would have access to diagnostic, medication, and medical clearance information. This practice could potentially reduce the time spent in emergency rooms and also reduce the testing performed in an emergency room before treatment admission. The ability of emergency room providers to access a patients' SUD treatment history could facilitate decisions on a treatment plan, potentially saving costs and time in emergency department settings.

3. Financial challenges: SUD treatment agencies are often strapped for funds, limiting their ability to spend on EHR infrastructure, initial training, maintenance, and user support. High rates of staff turnover and agency reorganization also increase training and infrastructure costs substantially. Furthermore, even if funds could be provided to incorporate harmonized EHR, long-term HIT support may not be feasible with current funding streams.

4. Deployment challenges: Implementing interoperable EHR in the context of creating a single source platform is formidable in many settings. First, security issues of traversing both inbound and outbound firewalls at different institutions could be problematic. Second, software standards from HIT organizations such as HL7, CDISC, IHE, and CCHIT involved in development and support of data standards attempting to enable interoperability evolve, require continuing maintenance. Third, if clinicians are typically paid by output, then when a new mandate comes for using harmonized EHR containing standardized parameters and core common elements relevant to SUD treatment, the ASAM PPC tool, ASI or other instruments may be seen as duplication. ${ }^{15,18,24,25}$

\section{Pathways to implementation}

In order to successfully develop and implement an interoperable EHR in behavioral health treatment settings (for primary care and specialized SUD treatment programs), one must adopt a multidisciplinary approach with outreach and buy-in from all stakeholders, including treatment providers, patients, payers, billing staff, administrators, policy makers, and vendors, to decide on a set of standardized core data elements to be included in harmonized systems. This multidisciplinary approach should tailor EHR development by providing flexibility in the design of EHR interfaces so that they may be modified to meet treatment agency needs to: (1) manage their daily work load; (2) be tailored to help 
the end-user agencies fill gaps in inefficient business and administrative processes; (3) address how to communicate health information across different reimbursement systems in a multi-payer system. In primary care settings, an imperative step is to incorporate brief, validated screeners in EHR that capture key information about SUD risk (including core questions on alcohol and tobacco use/misuse) while minimizing clinicians' assessment burden.

These EHR should employ common technical and semantic data standards and vocabularies around a brief core set of clinically relevant admission, treatment process, and discharge information to permit the above functions of EHR. Furthermore, achievable steps in primary care and specialized SUD treatment settings for reforming behavioral health data systems to incorporate interoperable EHR should include: (1) collecting encounter-level data (one record for each interaction between a provider and patient); (2) forging links and exchanges of information among primary care, SUD treatment, mental health, and state data systems having similar vocabulary/terminologies to streamline the delivery of care and enhance the coordination of health services; (3) investing in adaptable, flexible, nonproprietary open-source systems that foster system interoperability and facilitate the rapid adaptation of systems in response to changing agency and community needs. ${ }^{11,13,15}$ Evidence-based health care will benefit from the information available from interoperable EHR during patient care, and the utilization of the data collected to provide evidence of treatment success. ${ }^{11-13,15}$

To enhance buy-in from providers, payers, and other vital stakeholders, they should be informed of the Medicare and Medicaid payment incentives for eligible professionals who are meaningful EHR users, as is stipulated in the Health Information Technology for Economic and Clinical Health Act (or the HITECH Act) of The American Recovery and Reinvestment Act (ARRA). Through this Act, the current US administration called and provided substantive incentives for broad investment in development, adoption, and "meaningful use" of interoperable certified EHR across the country by the year 2015. In particular, ARRA sets aside billions of US Dollars in Medicare and Medicaid incentives administered by the Centers for Medicare and Medicaid Services for this purpose. ${ }^{23}$ The effort of establishing national data standards for these certified EHRs is headed at the federal level by the ONC. ${ }^{23}$ However, it remains to be seen whether the national data standards will contain standards and core common data elements relevant to SUD treatment. Thus, a key initiative has been for National Institute on Drug Abuse (NIDA) and SAMHSA, in coordination with ONC, to take the lead in working with relevant federal, state, community, and industry organization stakeholders to develop through a consultative iterative process a consensus on common data elements pertinent to SUD treatment (including questions on alcohol and tobacco use/misuse) to include in these interoperable EHRs. This initiative is important because these decisions regarding patient information to be included nationally in these standardized data systems and the EHR to be built based upon them may affect many aspects of SUD treatment delivery, research, and finance for many years to come. ${ }^{25}$

To this end, NIDA's Clinical Trials Network is currently supporting development of a brief set of core questions concerning the screening, clinical diagnosis, and treatment of SUD for incorporation into a national EHR. Eventually these common data elements will serve the needs of medical providers and healthcare planners and administrators in providing uniformity in entry and retrieval of health information in facilities across the nation. These SUD EHR questions will incorporate standards necessary to ensure semantic interoperability according to requirements for meaningful use of "certified EHR technology". Screening questions considered are published and validated singlequestion screeners for alcohol, tobacco, and illicit drug use, followed by brief, validated screening and assessment instruments for alcohol use disorders, tobacco use, and illicit drug use. Domains and sub-domains developed closely correspond to criteria in the ASAM PPC tool and JCAHO accreditation criteria. We are also planning to incorporate functionality with respect to physician-decision support for practice-based brief interventions and appropriate referral to specialty treatment, and to that end have begun consulting with active practices to identify the processes and logic used in the standard of care for interventions and treatment of SUD.

\section{Conclusion}

Development, implementation, and adoption of interoperable EHR containing standard vocabulary/terminologies and consensus-based common data elements is an essential means to achieve substantive national healthcare reform in the realm of SUD specialty treatment settings. Such HIT systems may not only reduce inefficient, duplicative, and/or uncoordinated healthcare service delivery but may also enable bridging the divide between specialized SUD 
treatment and mainstream medical care. Meaningful use of interoperable EHR may benefit patients, payers, and providers by reducing costs, improving quality of medical decision making and care, aiding in guideline implementation, and aiding in billing, reimbursement, and other administrative processes in clinical care. ${ }^{10-13,15}$ An interoperable EHR is also key to accelerating the translation of promising treatments from the bench to bedside to community-based practice settings. ${ }^{11,12,15}$ Recent federal initiatives through ARRA provide substantive financial incentives for healthcare providers and payers to develop and adopt such interoperable EHR. ${ }^{23}$ Since healthcare reimbursement systems (for example, Medicaid Management Information Systems) are moving toward integrated HIT systems with certified, standards-based EHR, providers who are unable to conform both claims and quantitative patient information to national data standards of these systems may become increasingly marginalized. ${ }^{25}$ Moreover, if specialty provider systems are unable to communicate both qualitative patient information and claims data in a manner consistent with these national standards, it is unlikely that the providers will be paid. ${ }^{25}$ Parity for SUD treatment is now federal law, but will be implemented in the context of these emerging EHR and national data standards. Therefore, specialty SUD treatment providers need to proactively initiate efforts to equip themselves with the necessary infrastructure to implement data standards consistent with those supported by the Federal Government. ${ }^{25}$ Efforts to develop consensusbased common data elements relevant to SUD treatment, which could be incorporated nationally into interoperable EHR, are key steps to ensure that SUD treatment is well integrated in this important comprehensive national public health initiative.

\section{Acknowledgments}

The authors thank Robert Lindblad, Robert Gore-Langton, and Kenneth $\mathrm{R}$ Gersing for critical reading and comments on this article.

\section{Disclosure}

The authors are employees of the Center for the Clinical Trials Network of the National Institute on Drug Abuse, National Institutes of Health, the funding agency for the National Drug Abuse Treatment Clinical Trials Network. The opinions in this manuscript are those of the authors and do not represent the official position of the US government.

\section{References}

1. Jha AK, DesRoches CM, Campbell EG, et al. Use of electronic health records in US hospitals. $N$ Engl J Med. 2009;360:1628-1638.

2. Shortliffe EH, Sondik EJ. The public health informatics infrastructure: anticipating its role in cancer. Cancer Causes Control. 2006;17: 861-869.

3. Burnam MA, Watkins KE. Substance abuse with mental disorders: specialized public systems and integrated care. Health Aff (Millwood). 2006;25:648-658.

4. Lee SY, Morrissey JP, Thomas KC, Carter WC, Ellis AR. Assessing the service linkages of substance abuse agencies with mental health and primary care organizations. Am J Drug Alcohol Abuse. 2006;32:69-86.

5. Lucado J, Paez K, Elixhauser A. Medication-Related Adverse Outcomes in US Hospitals and Emergency Departments, 2008. Vol. Statistical Brief \#109. http://mailview.custombriefings.com/mailview.aspx? $\mathrm{m}=2011041501$ dia\& $\mathrm{r}=2013302927$-c2011041159\&1=20110410222011041509c2011041507\&t=c, April, 2011. Accessed June 15, 2011: Agency for Healthcare Research and Quality (AHRQ).

6. SAMHSA DAWN Report. Trends in emergency department visits involving nonmedical use of narcotic pain relievers. http://store.samhsa. gov/product/DAWN10-0618. Accessed June 15, 2011: Substance Abuse and Mental Health Services Administration (SAMHSA); 2010.

7. Institute of Medicine. The computer-based patient record: an essential technology for health care, 1st ed. Washington, DC: National Academies Press; 1991.

8. National Library of Medicine. Biomedical Applications of High Performance Computing and Communications. BAA Available: NLM93-105/PSP. http://grants.nih.gov/grants/guide/notice-files/not93-126. html. NIH Guide. 1993;22(15). Accessed June 25, 2011.

9. Brinner KA, Downing GJ; American Health Information Community Personalized Health Care Workgroup. Advancing patient-centered pediatric care through health information exchange: update from the American Health Information Community Personalized Health Care Workgroup. Pediatrics. 2009;123(Suppl 2):S122-S124.

10. Institute of Medicine. Improving the quality of health care for mental and substance-use conditions. Washington, DC: National Academies Press; 2006.

11. Etheredge LM. A rapid-learning system. Health Aff (Millwood). 2007; 26:w107-w118.

12. Balas A, Al Sanousi A. Interoperable electronic patient records for health care improvement. Stud Health Technol Inform. 2009;150:19-23.

13. Institute of Medicine. Evidence-based medicine and the changing nature of health care. Washington, DC: The National Academies Press; 2008.

14. Goldstein MM, Blumenthal D. Building an information technology infrastructure. J Law Med Ethics. 2008;36:709-715.

15. Coffey RM, Buck JA, Kassed CA, et al. Transforming mental health and substance abuse data systems in the United States. Psychiatr Serv. 2008;59:1257-1263.

16. DesRoches CM, Campbell EG, Rao SR, et al. Electronic health records in ambulatory care - A National Survey of Physicians. N Engl J Med. 2008;359:350-360.

17. Wisdom JP, Ford JH 2nd, Hayes RA, Edmunson E, Hoffman K, McCarty D. Addiction treatment agencies' use of data: a qualitative assessment. J Behav Health Serv Res. 2006;33:394-407.

18. Wisdom JP, Ford JH 2nd, Wise M, Mackey D, Green CA. Substance abuse treatment programs' data management capacity: an exploratory study. J Behav Health Serv Res. 2011;38:249-264.

19. Popovits RM. Confidentiality law: time for change? Behav Healthc. 2010;30:11-13.

20. Wiley InterScience. 42 CFR Part 2 Update: sign-on letter to hyde points to need for federal facilitator. Alcoholism and Drug Abuse Weekly. 2010;22:6.

21. Dunlop BW. Should sensitive information from clinical trials be included in electronic medical records? JAMA. 2010;304:685-686. 
22. Salomon RM, Blackford JU, Rosenbloom ST, et al. Openness of patients' reporting with use of electronic records: psychiatric clinicians' views. J Am Med Inform Assoc. 2010;17:54-60.

23. Buntin MB, Jain SH, Blumenthal D. Health information technology: laying the infrastructure for national health reform. Health Aff (Millwood). 2010;29:1214-1219.

24. McLellan AT, Carise D, Kleber HD. Can the national addiction treatment infrastructure support the public's demand for quality care? J Subs Abuse Treat. 2003;25:117-121.
25. Wanser D, Rosenblum D. Where are the Treatment Providers and State Agencies in the Health Information Technology Planning Process? http://www.jointogether.org/news/yourturn/commentary/2010/whereare-the-treatment-providers.html. JoinTogether.org. June 1, 2010. Accessed June 24, 2011.

\section{Publish your work in this journal}

Substance Abuse and Rehabilitation is an international, peer-reviewed, open access journal publishing original research, case reports, editorials, reviews and commentaries on all areas of addiction and substance abuse and options for treatment and rehabilitation. The manuscrip management system is completely online and includes a very quick and fair peer-review system. Visit http://www.dovepress.com/testimonials. php to read real quotes from published authors.

\footnotetext{
Submit your manuscript here: http://www.dovepress.com/substance abuse-and-rehabilitation-journal
} 